\title{
METASTATIC PURE MUCINOUS BREAST CARCINOMA: A CASE REPORT
}

Mirella Laranjeira Nunes', Maria Carolina da Trindade Henriques Assunção², Pryscila Correia de Queiroz e Silva², Rossano Robério Fernandes Araújo², Bruno Pacheco Pereira², Ana Leide Guerra dos Santos², Vidianna Barbosa Sampaio², João Esberard de Vasconcelos Beltrão Neto²

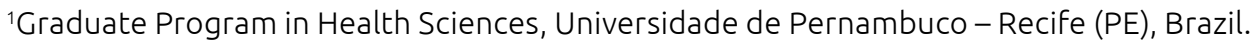

${ }^{2}$ Mastology Department, Oswaldo Cruz University Hospital-Recife (PE), Brazil.

Introduction: Pure mucinous breast carcinoma (PMBC) is rare, representing about $2 \%$ of breast cancers. Histologically, it is defined as having $90 \%$ or more of a mucinous component. It usually affects women between the ages of 55 and 60 years. Only $1 \%$ occurs in women below 35 years. It usually has positive hormone receptors (HRs); however, the positivity of HER-2 is rare. The management is not well established, being extrapolated from data on invasive ductal carcinoma. It usually presents a favorable prognosis, with rare lymph node (LN) involvement and metastasis rate of less than 15\%. HR status and nodal involvement are important prognostic factors. Case Report: A 34-year-old female arrived at the clinic with a tumor occupying all quadrants of the left breast for 5 months, along with skin thickening and hyperemia, hardened and enlarged left axillary LN. Ultrasonography showed a lesion with indistinct limits occupying almost the entire mammary parenchyma measuring $11.1 \times 12.8 \times 5.5 \mathrm{~cm}$, and the left LN enlarged in size, the largest measuring $3.1 \times 1.7 \mathrm{~cm}$. Bone scintigraphy and computed tomography (CT) showed suspicious metastatic lesion in the sternum, which could not be proven as metastasis due to the absence of structure to perform a biopsy. A clinical-prognostic staging IV, T4bN1Mx, was determined. Incisional biopsy diagnosed PMBC, histological grade 2. Immunohistochemistry results were HR positive, HER-2 positive (3+), and Ki67 70\%. The patient was treated chemotherapy drugs such as Adriamycin, cyclophosphamide, paclitaxel, and trastuzumab. Subsequently, a modified radical mastectomy was performed. The anatomopathology of the surgical specimen showed a complete pathological response. A new CT showed partial remission of sternal metastasis. Adjunctive treatment with trastuzumab, tamoxifen, radiotherapy, and surgical castration were performed. We brought an atypical case due to the presentation of PMBC in young woman, with a more aggressive pattern, with positive HER-2, metastasis, and complete pathological response with chemotherapy.

Keywords: Breast Neoplasm; Mucinous Adenocarcinoma; Neoplasm Metastasis. 